\title{
FREE FIELD REALIZATION OF VERTEX OPERATORS FOR LEVEL TWO MODULES OF $U_{q}(\widehat{\mathfrak{s l}}(2))$
}

\author{
YUJI HARA
}

\begin{abstract}
Free field realization of vertex operators for level two modules of $U_{q}(\widehat{\mathfrak{s l}}(2))$ are shown through the free field realization of the modules given by Idzumi in Ref. [4 [5]. We constructed types I and II vertex operators when the spin of the associated evaluation module is $1 / 2$ and type II's for the spin 1 .
\end{abstract}

\section{INTRODUCTION}

Vertex operators for the quantum affine algebra $U_{q}(\widehat{\mathfrak{s l}}(2))$ have played essential roles in the algebraic analysis of solvable lattice models since the pioneering works of [1, 2, [n]. In these works which analyze the XXZ model, type I vertex operators are identified with half infinite transfer matrices as their representation-theoretical counter part and type II vertex operators are interpreted as particle creation operators. To perform concrete computation such as a trace of composition of vertex operators, we need free field realization of modules and operators. In the said example of the XXZ model, the integral expressions of n-point correlation functions which are special cases of the traces are obtained through bosonization of level one module of $U_{q}(\widehat{\mathfrak{s l}}(2))$.

Motivated by these results, Idzumi 44, 5] constructed level two modules and type I vertex operators accompanied by spin 1 evaluation modules for $U_{q}(\widehat{\mathfrak{s l}}(2))$ in terms of bosons and fermions and then calculated correlation functions of a spin 1 analogue of the XXZ model. The purpose of this paper is to extend Idzumi's free field realization to other kinds of vertex operators i.e. type I and II vertex operators for the level two modules associated with the evalution module of spin $1 / 2$ and the type II's for the spin 1 . The results are given in Section 3 and their derivation is discussed in the first case in Section 4 . The results together with Ref. [4, 5] give the complete set of vertex operators for level two module of $U_{q}(\widehat{\mathfrak{s l}}(2))$ and enable one to calculate form factors of the spin 1 analogue of the XXZ model.

Recently Jimbo and Shiraishi [7] showed a coset-type construction for the deformed Virasoro algebra with the vertex operators for $U_{q}(\widehat{\mathfrak{s l}}(2))$. They constructed a primary operator for the deformed Virasoro algebra as coset type composition of vertex operators which may be denoted as $\left(U_{q}(\widehat{\mathfrak{s l}}(2))_{k} \oplus U_{q}(\widehat{\mathfrak{s l}}(2))_{1}\right) / U_{q}(\widehat{\mathfrak{s l}}(2))_{k+1}$. We hope that our results will be helpful 
for extending this work to the deformed supersymmetric Virasoro algebra through $\left(U_{q}(\widehat{\mathfrak{s} l}(2))_{k} \oplus\right.$ $\left.U_{q}(\widehat{\mathfrak{s l}}(2))_{2}\right) / U_{q}(\widehat{\mathfrak{s l}}(2))_{k+2}$.

\section{Free field REALization of LeVel two module}

2.1. Convention. In the following we will use $U$ to denote the quantum affine algebra $U_{q}(\widehat{\mathfrak{s l}}(2))$. Unless mentioned, we follow the notations of Ref. 橉, 5. As for the free field representation, we slightly modify the convention.

The quantum affine algebra $U$ is an associative algebra with unit 1 generated by $e_{i}, f_{i}(i=$ $0,1), q^{h}\left(h \in P^{*}\right)$ with relations

$$
\begin{gathered}
q^{0}=1, q^{h} q^{h^{\prime}}=q^{h+h^{\prime}}, \\
q^{h} e_{i} q^{-h}=q^{\left\langle h, \alpha_{i}\right\rangle} e_{i}, q^{h} f_{i} q^{-h}=q^{-\left\langle h, \alpha_{i}\right\rangle} f_{i}, \\
{\left[e_{i}, f_{i}\right] \text { 国 }=\delta_{i j} \frac{t_{i}-t_{i}^{-1}}{q-q^{-1}}, \quad\left(t_{i}=q^{h_{i}}\right)} \\
e_{i}^{3} e_{j}-[3] e_{i}^{2} e_{j} e_{i}+[3] e_{i} e_{j} e_{i}^{2}-e_{j} e_{i}^{3}=0, \\
f_{i}^{3} f_{j}-[3] f_{i}^{2} f_{j} f_{i}+[3] f_{i} f_{j} f_{i}^{2}-f_{j} f_{i}^{3}=0,
\end{gathered}
$$

where $P=\mathbb{Z} \Lambda_{0}+\mathbb{Z} \Lambda_{1}+\mathbb{Z} \delta$ is the weight lattice of the affine Lie algebra $\widehat{\mathfrak{s} l}(2)$ and $P^{*}$ is the dual lattice to $P$ with the dual basis $\left\{h_{0}, h_{1}, d\right\}$ to $\left\{\Lambda_{0}, \Lambda_{1}, \delta\right\}$ with respect to the natural pairing $\langle\rangle:, P \times P^{*} \rightarrow \mathbb{Z}$. We also use current type generators introduced by Drinfeld [11]

$$
\begin{gathered}
{\left[a_{k} \cdot a_{l}\right]=\delta_{k+l, 0} \frac{[2 k]}{k} \frac{\gamma^{k}-\gamma^{-k}}{q-q^{-1}},} \\
K a_{k} K^{-1}=a_{k}, K x_{k}^{ \pm} K^{-1}=q^{ \pm 2} x_{k}^{ \pm}, \\
{\left[a_{k}, x_{l}^{ \pm}\right]= \pm \frac{[2 k]}{k} \gamma^{\mp|k| / 2} x_{k+l}^{ \pm},} \\
x_{k+l}^{ \pm} x_{l}^{ \pm}-q^{ \pm 2} x_{l}^{ \pm} x_{k+l}^{ \pm}=q^{ \pm 2} x_{k}^{ \pm} x_{l+1}^{ \pm}-x_{l+1}^{ \pm} x_{k}^{ \pm}, \\
{\left[x_{k}^{+}, x_{l}^{-}\right]=\frac{\gamma^{\frac{k-l}{2}} \psi_{k+l}-\gamma^{\frac{l-k}{2}} \phi_{k+l}}{q-q^{-1}},}
\end{gathered}
$$

where $\psi_{k}$, and $\varphi_{k}$ are defined as

$$
\sum_{k \geqslant 0} \psi_{k} z^{-k}=K \exp \left\{\left(q-q^{-1}\right) \sum_{k \geqslant 1} a_{k} z^{-k}\right\}
$$

$*[\mathrm{~A}, \mathrm{~B}]=\mathrm{AB}-\mathrm{BA}$ 


$$
\sum_{k \geqslant 0} \phi_{k} z^{k}=K^{-1} \exp \left\{-\left(q-q^{-1}\right) \sum_{k \geqslant 1} a_{-k} z^{k}\right\} .
$$

The relation between two types of generators are

$$
t_{1}=K, t_{0}=\gamma K^{-1}, e_{1}=x_{0}^{+}, e_{0} t_{1}=x_{1}^{-}, f_{1}=x_{0}^{-}, t_{1}^{-1} f_{1}=x_{0}^{-1} .
$$

The higest weight module and the evaluation module are described compactly in Ref. 迎.

Commutation and anti commutation relations of bosons and fermions are given by

$$
\begin{gathered}
{\left[a_{m}, a_{n}\right]=\delta_{m+n, 0} \frac{[2 m]^{2}}{m},} \\
\left\{\phi_{m}, \phi_{n}\right\}=\delta_{m+n, 0} \eta_{m}, \\
\eta_{m}=q^{2 m}+q^{-2 m} .
\end{gathered}
$$

where $m, n \in \mathbb{Z}+1 / 2$ or $\in \mathbb{Z}$ for Neveu-Schwarz-sector or Ramond-sector respectively. Fock spaces and vacuum vectors are denoted as $\mathcal{F}^{a}, \mathcal{F}^{\phi^{N S}}, \mathcal{F}^{\phi^{R}}$ and $|v a c\rangle,|N S\rangle,|R\rangle$ for the boson and $N S$ and $R$ fermion respectively. Fermion currents are defined as

$$
\phi^{N S}(z)=\sum_{n \in \mathbb{Z}+\frac{1}{2}} \phi_{n}^{N S} z^{-n}, \phi^{R}(z)=\sum_{n \in \mathbb{Z}} \phi_{n}^{R} z^{-n} .
$$

$Q=\mathbb{Z} \alpha$ is the root lattice of $\mathfrak{s l}_{2}$ and $F[Q]$ be the group algebra. We use $\partial$ as

$$
[\partial, \alpha]=2 .
$$

2.2. $V\left(2 \Lambda_{0}\right), V\left(2 \Lambda_{1}\right)$. The highest weight module $V\left(2 \Lambda_{0}\right)$ is identified with the Fock space

$$
\mathcal{F}_{+}^{(0)}=\mathcal{F}^{a} \otimes\left\{\left(\mathcal{F}_{\text {even }}^{\phi^{N S}} \otimes F[2 Q]\right) \oplus\left(\mathcal{F}_{\text {odd }}^{\phi^{N S}} \otimes e^{\alpha} F[2 Q]\right)\right\}
$$

subscripts even and odd represent the number of fermions. The highest weight vector is $|v a c\rangle \otimes$ $|N S\rangle \otimes 1 . V\left(2 \Lambda_{1}\right)$ is

$$
\mathcal{F}_{-}^{(0)}=\mathcal{F}^{a} \otimes\left\{\left(\mathcal{F}_{\text {even }}^{\phi^{N S}} \otimes e^{\alpha} F[2 Q]\right) \oplus\left(\mathcal{F}_{\text {odd }}^{\phi^{N S}} \otimes F[2 Q]\right)\right\}
$$

with the highest weight vector being $|v a c\rangle \otimes|N S\rangle \otimes e^{\alpha}$. Note that

$$
\begin{gathered}
\mathcal{F}^{(0)}=\mathcal{F}_{-}^{(0)} \oplus \mathcal{F}_{+}^{(0)} . \\
\mathcal{F}^{(0)}=\mathcal{F}^{a} \otimes \mathcal{F}^{\phi^{N S}} \otimes F[Q] .
\end{gathered}
$$

The operators are realized in the following manner.

$$
\begin{gathered}
\gamma=q^{2}, \quad K=q^{\partial} \\
x^{ \pm}(z)=\sum_{m \in \mathbb{Z}} x_{m}^{ \pm} z^{-m}=E_{<}^{ \pm}(z) E_{>}^{ \pm}(z) \phi^{N S}(z) e^{ \pm \alpha} z^{\frac{1}{2} \pm \frac{1}{2} \partial},
\end{gathered}
$$

\footnotetext{
$*\{\mathrm{~A}, \mathrm{~B}\}=\mathrm{AB}-\mathrm{BA}$
} 


$$
E_{<}^{ \pm}(z)=\exp \left( \pm \sum_{m>0} \frac{a_{-m}}{[2 m]} q^{\mp m} z^{m}\right), \quad E_{>}^{ \pm}(z)=\exp \left(\mp \sum_{m>0} \frac{a_{m}}{[2 m]} q^{\mp m} z^{-m}\right)
$$

and

$$
\begin{gathered}
d=-\frac{\partial^{2}}{8}+\frac{(\lambda, \lambda)}{4}-\sum_{m=1}^{\infty} m N_{m}^{a}-\sum_{k>0} k N_{k}^{\phi^{N S}} \\
N_{m}^{a}=\frac{m}{[2 m]^{2}} a_{-m} a_{m}, \quad N_{k}^{\phi^{N S}}=\frac{1}{\eta_{m}} \phi_{-m}^{N S} \phi_{m}^{N S} \quad(m>0),
\end{gathered}
$$

where the higest weight vector of the module should be substituted for $\lambda$ of (3).

2.3. $V\left(\Lambda_{0}+\Lambda_{1}\right)$. The module $V\left(\Lambda_{0}+\Lambda_{1}\right)$ is identified with

$$
\mathcal{F}^{(1)}=\mathcal{F}^{a} \otimes \mathcal{F}^{\phi^{R}} \otimes e^{\frac{\alpha}{2}} F[Q],
$$

where

$$
\phi_{0}^{R}|R\rangle=|R\rangle
$$

The highest weight vector is identified with $|v a c\rangle \otimes|R\rangle \otimes e^{\frac{\alpha}{2}}$.

Operators are constructed in the same way as before except that subscripts for fermion sector are $R$ instead of $N S$.

\section{FreE FiELD REALizATiONS OF VERTEX OPERATORS}

Let $V, V^{\prime}$ be level two modules and $V_{z}^{(k)}$ be a spin $\mathrm{k} / 2$ evaluation module of $U$. Vertex operators we will consider are $U$-linear maps of the following kinds [8, 9]

$$
\begin{gathered}
\Phi_{V}^{V^{\prime}, k}(z): V \longrightarrow V^{\prime} \otimes V_{z}^{(k)} \\
\Psi_{V}^{k, V^{\prime}}(z): V \longrightarrow V_{z}^{(k)} \otimes V^{\prime} .
\end{gathered}
$$

Vertex operators of the form (6, (6) are called type I and II respectively. Components of vertex operators are defined as

$$
\Phi(z)_{V}^{V^{\prime}, k}=\sum_{n=0}^{k} \Phi_{n}(z) \otimes u_{n}, \Psi(z)_{V}^{k, V^{\prime}}=\sum_{n=0}^{k} u_{n} \otimes \Psi_{n}(z) .
$$

3.1. type I Vertex Operators for level 2 and spin 1/2. We show free field realization of type I vertex operators of the following kinds

$$
\begin{gathered}
\Phi_{2 \Lambda_{i}}^{\Lambda_{0}+\Lambda_{1}, 1}(z): V\left(2 \Lambda_{i}\right) \longrightarrow V\left(\Lambda_{0}+\Lambda_{1}\right) \otimes V_{z}^{(1)}, \\
\Phi_{\Lambda_{0}+\Lambda_{1}}^{2 \Lambda_{i}, 1}(z): V\left(\Lambda_{0}+\Lambda_{1}\right) \longrightarrow V\left(2 \Lambda_{i}\right) \otimes V_{z}^{(1)}
\end{gathered}
$$

where $i=0$ or 1 . 
Under the free field realization of level 2 modules reviewed in Secton 2, the explicit forms of the components of the vertex operators in (8) are

$$
\begin{gathered}
\Phi_{1}(z)=B_{I,<}(z) B_{I,>}(z) \Omega_{N S}^{R}(z) e^{\alpha / 2}\left(-q^{4} z\right)^{\partial / 4} \\
\Phi_{0}(z)=\oint \frac{d w}{2 \pi i} B_{I,<}(z) E_{<}^{-}(w) B_{I,>}(z) E_{>}^{-}(w) \Omega_{N S}^{R}(z) \phi^{N S}(w) \\
\times e^{-\alpha / 2}\left(-q^{4} z\right)^{\partial / 4} w^{-\partial / 2}\left(-q^{4} z w^{3}\right)^{-\frac{1}{2}} \frac{\left(\frac{w}{q^{3} z} ; q^{4}\right)_{\infty}}{\left(\frac{w}{q z} ; q^{4}\right)_{\infty}}\left\{\frac{w}{1-q^{-3} w / z}+\frac{q^{5} z}{1-q^{5} z / w}\right\}, \\
B_{I,<}(z)=\exp \left(\sum_{n=1}^{\infty} \frac{[n] a_{-n}}{[2 n]^{2}}\left(q^{5} z\right)^{n}\right) \\
B_{I,>}(z)=\exp \left(-\sum_{n=1}^{\infty} \frac{[n] a_{n}}{[2 n]^{2}}\left(q^{3} z\right)^{-n}\right) .
\end{gathered}
$$

The integrand of $\Phi_{0}(z)$ has poles only at $w=q^{5} z, q^{3} z$ except for $w=0, \infty$ and the contour of integration encloses $w=0, q^{5} z$, details are discussed in Sec. 4 . For those of (9) we just replace $\Omega_{N S}^{R}(z)$ with $\Omega_{R}^{N S}(z)$ in $(10,11)$.

The fermionic part $\Omega(z)$ 's are maps between different fermion sectors and satisfy

$$
\phi^{N S}(w) \Omega(z)_{R}^{N S}=\left(\frac{-q^{4} z}{w}\right)^{1 / 2} \frac{\left(\frac{w}{q^{3} z} ; q^{4}\right)_{\infty}\left(\frac{q^{7} z}{w} ; q^{4}\right)_{\infty}}{\left(\frac{w}{q z} ; q^{4}\right)_{\infty}\left(\frac{q^{5} z}{w} ; q^{4}\right)_{\infty}} \Omega(z)_{R}^{N S} \phi^{R}(w)
$$

and exactly the same equation except subscripts for fermion sectors are exchanged. This kind of mapping for fermions first appeared in high-energy phisics theory as "fermion emission vertex operator" [6, 10]. Their free field realizations are

$$
\begin{gathered}
\Omega_{N S}^{R}(z)=\left\langle N S\left|e^{Y}\right| R\right\rangle \\
Y=-\sum_{m>n \geq 0} X_{m, n} \varphi_{-m}^{R} \varphi_{-n}^{R} z^{m+n}-\sum_{k>l \geq 0} X_{k+1 / 2, l+1 / 2} \varphi_{k+1 / 2}^{N S} \varphi_{l+1 / 2}^{N S} z^{-k-l-1} \\
+\sum_{\substack{m \geq 0 \\
k \geq 0}} X_{m,-k-1 / 2} \varphi_{-m}^{R} \varphi_{k+1 / 2}^{N S} z^{m-k-1 / 2}
\end{gathered}
$$




$$
\begin{gathered}
\Omega_{R}^{N S}(z)=\left\langle R\left|e^{Y^{\prime}}\right| N S\right\rangle \\
Y^{\prime}=\sum_{k>l \geq 0} X_{k+1 / 2, l+1 / 2} \varphi_{-k-1 / 2}^{N S} \varphi_{-l-1 / 2}^{N S} z^{k+l+1}+\sum_{m>n \geq 0} X_{m, n} \varphi_{m}^{R} \varphi_{n}^{R} z^{-m-n} \\
-\sum_{\substack{k \geq 0 \\
m \geq 0}} X_{-k-1 / 2, m} \varphi_{-k-1 / 2}^{N S} \varphi_{m}^{R} z^{k-m+1 / 2} \\
\varphi_{0}^{R}=\phi_{0}^{R}, \quad \varphi_{-m}^{R}=\phi_{-m}^{R} \frac{\gamma_{m} q^{5 m}}{\eta_{m}}, \quad \varphi_{m}^{R}=\phi_{m}^{R} \frac{\gamma_{m} q^{-3 m}}{\eta_{m}} \quad(m>0), \quad(k>0), \\
\varphi_{k+1 / 2}^{N S}=\phi_{k+1 / 2}^{N S} \frac{\gamma_{k} q^{-3 k-2}}{\eta_{k+1 / 2}}\left(-(-1)^{1 / 2}\right), \quad \varphi_{-k-1 / 2}^{N S}=\phi_{-k-1 / 2}^{N S} \frac{\gamma_{k} q^{5 k+2}}{\eta_{k+1 / 2}}(-1)^{1 / 2} \quad\left(k>q_{k, l}=\frac{q^{4 k}-q^{4 l}}{1-q^{4(k+l)}},\right. \\
\gamma_{n}=\frac{\left(q^{2} ; q^{4}\right)_{n}}{\left(q^{4} ; q^{4}\right)_{n}}, \quad \frac{\left(q^{2} z ; q^{4}\right)_{\infty}}{\left(z ; q^{4}\right)_{\infty}}=\sum_{n=0}^{\infty} \gamma_{n} z^{n} .
\end{gathered}
$$

(15.17) are to mean that a matrix element is given by

$$
\begin{gathered}
{ }_{R}\left\langle\text { out }\left|\Omega_{N S}^{R}(z)\right| \text { in }\right\rangle_{N S}={ }_{R}\left\langle\text { out }\left|\otimes\left\langle N S\left|e^{Y}\right| R\right\rangle \otimes\right| \text { in }\right\rangle_{N S} \\
\text { for } \left.\mid \text { out }\rangle_{R} \in \mathcal{F}^{\phi^{R}}, \mid \text { in }\right\rangle_{N S} \in \mathcal{F}^{\phi^{N S}}
\end{gathered}
$$

We define the normalized vertex operators $\tilde{\Phi}(z)$ 's as follows

$$
\begin{array}{ll}
\left\langle\Lambda_{0}+\Lambda_{1}\left|\tilde{\Phi}_{1}(z)\right| 2 \Lambda_{0}\right\rangle=1, & \left\langle 2 \Lambda_{1}\left|\tilde{\Phi}_{1}(z)\right| \Lambda_{0}+\Lambda_{1}\right\rangle=1, \\
\left\langle\Lambda_{0}+\Lambda_{1}\left|\tilde{\Phi}_{0}(z)\right| 2 \Lambda_{1}\right\rangle=1, & \left\langle 2 \Lambda_{0}\left|\tilde{\Phi}_{0}(z)\right| \Lambda_{0}+\Lambda_{1}\right\rangle=1,
\end{array}
$$

and these are given by

$$
\begin{gathered}
\tilde{\Phi}_{2 \Lambda_{0}}^{\Lambda_{0}+\Lambda_{1}, 1}(z)=\Phi(z), \\
\tilde{\Phi}_{\Lambda_{0}+\Lambda_{1}}^{2 \Lambda_{1}, 1}(z)=\left(-q^{4} z\right)^{-1 / 4} \Phi(z), \\
\tilde{\Phi}_{\Lambda_{0}+\Lambda_{1}}^{2 \Lambda_{0}, 1}(z)=\left(-q^{4} z\right)^{1 / 4} \Phi(z), \\
\tilde{\Phi}_{2 \Lambda_{1}}^{\Lambda_{0}+\Lambda_{1}, 1}(z)=\left(-q^{6} z\right)^{-1 / 2} \Phi(z) .
\end{gathered}
$$

3.2. type II Vertex Operators for level 2 and spin 1/2. We consider type II vertex operators of the following kind

$$
\begin{gathered}
\Psi_{2 \Lambda_{i}}^{1, \Lambda_{0}+\Lambda_{1}}(z): V\left(2 \Lambda_{i}\right) \longrightarrow V_{z}^{(1)} \otimes V\left(\Lambda_{0}+\Lambda_{1}\right), \\
\Psi_{\Lambda_{0}+\Lambda_{1}}^{1,2 \Lambda_{i}}(z): V\left(\Lambda_{0}+\Lambda_{1}\right) \longrightarrow V_{z}^{(1)} \otimes V\left(2 \Lambda_{i}\right) .
\end{gathered}
$$


Explicit forms of the components are as follows.

$$
\begin{gathered}
\Psi_{0}(z)=B_{I I,<}(z) B_{I I,>}(z) \Omega\left(q^{-2} z\right) e^{-\alpha / 2}\left(-q^{2} z\right)^{-\partial / 4} \\
\Psi_{1}(z)=\oint \frac{d w}{2 \pi i} B_{I I,<}(z) E_{<}^{+}(w) B_{I I,>}(z) E_{>}^{+}(w) \Omega\left(q^{-2} z\right) \phi(w) \\
\times e^{\alpha / 2}\left(-q^{2} z\right)^{-\partial / 4} w^{\partial / 2}\left(-q^{2} z w^{3}\right)^{-\frac{1}{2}} \frac{\left(\frac{w}{q z} ; q^{4}\right)_{\infty}}{\left(\frac{q w}{z} ; q^{4}\right)_{\infty}}\left\{\frac{w}{1-q^{-3} w / z}+\frac{q^{3} z}{1-q z / w}\right\}, \\
B_{I I,<}(z)=\exp \left(-\sum_{n=1}^{\infty} \frac{[n] a_{-n}}{[2 n]^{2}}(q z)^{n}\right) \\
B_{I I,>}(z)=\exp \left(\sum_{n=1}^{\infty} \frac{[n] a_{n}}{[2 n]^{2}}\left(q^{3} z\right)^{-n}\right) .
\end{gathered}
$$

The integrand of $\Psi_{1}(z)$ has poles only at $w=q^{3} z, q z$ except for $w=0, \infty$ and the contour of integration encloses $w=0, q z$. Subscripts for fermion sectors are abbreviated.

Normalized vertex operators are defined by the conditions

$$
\begin{array}{ll}
\left\langle\Lambda_{0}+\Lambda_{1}\left|\tilde{\Psi}_{1}(z)\right| 2 \Lambda_{0}\right\rangle=1, & \left\langle 2 \Lambda_{1}\left|\tilde{\Psi}_{1}(z)\right| \Lambda_{0}+\Lambda_{1}\right\rangle=1 \\
\left\langle\Lambda_{0}+\Lambda_{1}\left|\tilde{\Psi}_{0}(z)\right| 2 \Lambda_{1}\right\rangle=1, & \left\langle 2 \Lambda_{0}\left|\tilde{\Psi}_{0}(z)\right| \Lambda_{0}+\Lambda_{1}\right\rangle=1
\end{array}
$$

and these are given by

$$
\begin{gathered}
\tilde{\Psi}_{2 \Lambda_{0}}^{1, \Lambda_{0}+\Lambda_{1}}(z)=(-q)^{-1} \Psi(z), \\
\tilde{\Psi}_{\Lambda_{0}+\Lambda_{1}}^{1,2 \Lambda_{1}}(z)=-\left(-q^{6} z\right)^{-1 / 4} \Psi(z), \\
\tilde{\Psi}_{\Lambda_{0}+\Lambda_{1}}^{1,2 \Lambda_{0}}(z)=\left(-q^{2} z\right)^{1 / 4} \Psi(z), \\
\tilde{\Psi}_{2 \Lambda_{1}}^{1, \Lambda_{0}+\Lambda_{1}}(z)=\left(-q^{2} z\right)^{1 / 2} \Psi(z) .
\end{gathered}
$$

3.3. type II Vertex Operators for level 2 and spin 1. When the spin of the evaluation module is 1 , the type II vertex operators do not contain any fermion emission vertex operators.

$$
\begin{aligned}
\Psi_{2 \Lambda_{i}}^{2,2 \Lambda_{i}}(z) & : V\left(2 \Lambda_{i}\right) \longrightarrow V_{z}^{(2)} \otimes V\left(2 \Lambda_{i}\right), \\
\Psi_{\Lambda_{0}+\Lambda_{1}}^{2, \Lambda_{0}+\Lambda_{1}}(z) & : V\left(\Lambda_{0}+\Lambda_{1}\right) \longrightarrow V_{z}^{(2)} \otimes V\left(\Lambda_{0}+\Lambda_{1}\right) .
\end{aligned}
$$

Explicit form of the components are as follows.

$$
\begin{aligned}
\Psi_{0}(z) & =F_{I I,<}(z) F_{I I,>}(z) e^{-\alpha}\left(-q^{2} z\right)^{-\partial / 2+1} \\
\Psi_{1}(z) & =\oint \frac{d w}{2 \pi i} F_{I I,<}(z) E_{<}^{+}(w) F_{I I,>}(z) E_{>}^{+}(w) \phi(w)\left(\frac{w}{-q^{2} z}\right)^{\partial / 2} \\
& \times w^{-1 / 2}\left\{\frac{1}{1-\frac{w}{q^{4} z}}+\frac{q^{4} z}{w\left(1-\frac{z}{w}\right)}\right\},
\end{aligned}
$$


The integration contour encircles poles $w=0, z$ but the pole $w=q^{4} z$ lies outside of it.

$$
\begin{aligned}
\Psi_{2}(z)=\oint \frac{d w_{2}}{2 \pi i} & \oint \frac{d w_{1}}{2 \pi i} F_{I I,<}(z) E_{<}^{+}\left(w_{1}\right) E_{<}^{+}\left(w_{2}\right) F_{I I,>}(z) E_{>}^{+}\left(w_{1}\right) E_{>}^{+}\left(w_{2}\right) \\
& \times e^{\alpha}\left(\frac{w_{1} w_{2}}{-q^{2} z}\right)^{\partial / 2}\left(w_{1} w_{2}\right)^{-1 / 2}\left\{\frac{1}{1-\frac{w_{1}}{q^{4} z}}+\frac{q^{4} z}{w_{1}\left(1-\frac{z}{w_{1}}\right)}\right\} \\
& \times\left\{[2]^{-1}: \phi\left(w_{1}\right) \phi\left(w_{2}\right):\left(\frac{w_{1}-q^{-2} w_{2}}{-q^{2} z\left(1-\frac{w_{2}}{q^{4} w_{1}}\right)}+\frac{1-\frac{w_{1}}{q^{2} w_{2}}}{1-\frac{z}{w_{2}}}\right)\right. \\
& \left.+\frac{\left(w_{1} w_{2}\right)^{1 / 2}\left(1-\frac{w_{2}}{w_{1}}\right)}{-q^{2} z\left(1-\frac{w^{2} w_{2}}{w_{1}}\right)\left(1-\frac{w_{2}}{q^{4} z}\right)}-\frac{\left(1-\frac{w_{1}}{w_{2}}\right)}{\left(1-\frac{q^{2} w_{1}}{w_{2}}\right)\left(1-\frac{z}{w_{2}}\right)}\right\},
\end{aligned}
$$

We have to prepare two contours because of the fermionic part and one is for the term including $: \phi\left(w_{1}\right) \phi\left(w_{2}\right)$ : and the other is for the rest. The former satisfies $\left|\frac{w_{2}}{q^{4} w_{1}}\right|<1,\left|w_{2}\right|>|z|$ and the same condition satisfied by the contour for $P s i_{1}$ with substitution $w=w_{1}$. The latter satisfies $\left|q^{2} w_{2}\right|<\left|w_{1}\right|<\left|q^{-2} w_{2}\right|$ and the same conditions as $P s i_{1}$ with $w=w_{1}, w_{2}$.

$$
\begin{aligned}
& F_{I I,<}(z)=\exp \left(-\sum_{m>0} \frac{a_{-m}}{[2 m]}(q z)^{m}\right), \\
& F_{I I,>}(z)=\exp \left(\sum_{m>0} \frac{a_{m}}{[2 m]}\left(q^{3} z\right)^{-m}\right) .
\end{aligned}
$$

Under the normailzation

$$
\begin{gathered}
\left\langle 2 \Lambda_{0}\left|\tilde{\Psi}_{0}(z)\right| 2 \Lambda_{1}\right\rangle=1, \quad\left\langle 2 \Lambda_{1}\left|\tilde{\Psi}_{2}(z)\right| 2 \Lambda_{0}\right\rangle=1 \\
\left\langle\Lambda_{0}+\Lambda_{1}\left|\tilde{\Psi}_{1}(z)\right| \Lambda_{0}+\Lambda_{1}\right\rangle=1
\end{gathered}
$$

$\tilde{\Psi}(z)^{\prime}$ are given by

$$
\begin{gathered}
\tilde{\Psi}_{2 \Lambda_{1}}^{2,2 \Lambda_{0}}(z)=\Psi(z), \\
\tilde{\Psi}_{\Lambda_{0}+\Lambda_{1}}^{2, \Lambda_{0}+\Lambda_{1}}(z)=-\left(-q^{2} z\right)^{-1 / 2} \Psi(z), \\
\tilde{\Psi}_{2 \Lambda_{0}}^{2,2 \Lambda_{1}}(z)=\left(-q^{4} z\right)^{-1} \Psi(z) .
\end{gathered}
$$

\section{Derivation}

Taking $\Phi_{2 \Lambda_{i}}^{\Lambda_{0}+\Lambda_{1}, 1}(z)$ as an example, we discuss the derivation of the results in the previous section. Other cases can be treated in almost the same way.

\subsection{General structure of $\Phi_{0}(z)$ and $\Phi_{1}(z)$. Calculating}

$$
\Delta(x) \Phi(z)=\Phi(z) x
$$


for $x=$ Chevalley generators of $U$ and $a_{n}$, we get

$$
\begin{gathered}
0=\left[\Phi_{1}(z), x_{0}^{+}\right], \\
K \Phi_{1}(z)=\left[\Phi_{0}(z), x_{0}^{+}\right], \\
0=x_{0}^{-} \Phi_{0}(z)-q \Phi_{0}(z) x_{0}^{-}, \\
\Phi_{0}(z)=\Phi_{1}(z) x_{0}^{-}-q x_{0}^{-} \Phi_{1}(z), \\
0=\Phi_{0}(z) x_{1}^{-}-q x_{1}^{-} \Phi_{0}(z), \\
q^{3} z \Phi_{0}(z)=\Phi_{1}(z) x_{1}^{-}-q^{-1} x_{1}^{-} \Phi_{1}(z), \\
(q z K)^{-1} \Phi_{1}(z)=\left[\Phi_{0}(z), x_{-1}^{+}\right], \\
0=\left[\Phi_{1}(z), x_{-1}^{+}\right], \\
K \Phi_{1}(z) K^{-1}=q \Phi_{1}(z), \\
K \Phi_{0}(z) K^{-1}=q^{-1} \Phi_{0}(z), \\
{\left[a_{m}, \Phi_{1}(z)\right]=\left(q^{5} z\right)^{m} \frac{[m]}{m} \Phi_{1}(z),} \\
{\left[a_{-m}, \Phi_{1}(z)\right]=\left(q^{3} z\right)^{-m} \frac{[m]}{m} \Phi_{1}(z) .}
\end{gathered}
$$

From (48,49,50), we can speculate the form of $\Phi_{1}(z)$ as

$$
\Phi_{1}(z)=B_{I,<}(z) B_{I,>}(z) \Omega_{N S}^{R}(z) e^{\alpha / 2} y^{\partial} .
$$

To determine $y$ and the fermionic part $\Omega_{N S}^{R}(z)$, we impose the following conditions on $\Phi_{1}(z)$

$$
\begin{aligned}
\Phi_{1}(z) x_{0}^{-}-q x_{0}^{-} \Phi_{1}(z) & =\left(q^{3} z\right)^{-1}\left(\Phi_{1}(z) x_{1}^{-}-q^{-1} x_{1}^{-} \Phi_{1}(z)\right), \\
0 & =\left[\Phi_{1}(z), x^{+}(w)\right]
\end{aligned}
$$

which can be easily seen from $(46,47)$ and the proposition of Section 4.4 of Ref.[12]. Then we have (10,14)

$$
\begin{gathered}
\Phi_{1}(z)=B_{I,<}(z) B_{I,>}(z) \Omega_{N S}^{R}(z) e^{\alpha / 2}\left(-q^{4} z\right)^{\partial / 4} \\
\phi^{R}(w) \Omega_{N S}^{R}(z)=\left(\frac{-q^{4} z}{w}\right)^{1 / 2} \frac{\left(\frac{w}{q^{3} z} ; q^{4}\right)_{\infty}\left(\frac{q^{7} z}{w} ; q^{4}\right)_{\infty}}{\left(\frac{w}{q z} ; q^{4}\right)_{\infty}\left(\frac{q^{5} z}{w} ; q^{4}\right)_{\infty}} \Omega_{N S}^{R}(z) \phi^{N S}(w) .
\end{gathered}
$$


$\Phi_{1}(z)$ can be calculated through 46

$$
\begin{aligned}
\Phi_{0}(z) & =\oint \frac{d w}{2 \pi i} \frac{1}{w}\left\{\Phi_{1}(z) x^{-}(w)-q x^{-}(w) \Phi_{1}(z)\right\} \\
& =\oint \frac{d w}{2 \pi i} B_{I,<}(z) E_{<}^{-}(w) B_{I,>}(z) E_{>}^{-}(w) \Omega_{N S}^{R}(z) \phi^{N S}(w) \\
& \times e^{-\alpha / 2}\left(-q^{4} z\right)^{\partial / 4} w^{-\partial / 2}\left(-q^{4} z w^{3}\right)^{-\frac{1}{2}} \frac{\left(\frac{w}{q^{3} z} ; q^{4}\right)_{\infty}}{\left(\frac{w}{q z} ; q^{4}\right)_{\infty}}\left\{\frac{w}{1-q^{-3} w / z}+\frac{q^{5} z}{1-q^{5} z / w}\right\},
\end{aligned}
$$

To determine the contour of integration we have to find the poles of $\Omega_{N S}^{R}(z) \phi^{N S}(w)$ and this can be seen from

$$
\left\langle R\left|\Omega_{N S}^{R}(z) \phi^{N S}(w)\right| N S\right\rangle=\frac{\left(\frac{w}{q z} ; q^{4}\right)_{\infty}}{\left(\frac{w}{q^{3} z} ; q^{4}\right)_{\infty}}, \quad\left\langle N S\left|\Omega_{R}^{N S}(z) \phi^{R}(w)\right| R\right\rangle=\left(\frac{w}{-q^{4} z}\right)^{1 / 2} \frac{\left(\frac{w}{q z} ; q^{4}\right)_{\infty}}{\left(\frac{w}{q^{3} z} ; q^{4}\right)_{\infty}} .
$$

Hence as a composite $\Omega_{N S}^{R}(z) \phi^{N S}(w) \frac{\left(\frac{w}{q^{3} z} ; q^{4}\right)_{\infty}}{\left(\frac{w}{q z} ; q^{4}\right)_{\infty}}$ in the integrand has no poles and the contour is the one encloses $w=0, q^{5} z$.

4.2. Fermion emission vertex operator. In Ref. [6], Eqn.(15) appears in the study of the Ising model and its free field realization is given without any details. Thus we give the exposition of its derivation?. The main point of derivating free field realization of the fermion emission vertex operator $\Omega_{N S}^{R}(z)(15,16)$ is to expand $\Omega_{N S}^{R}(z)$ as

$$
\begin{gathered}
\Omega_{N S}^{R}(z)=\sum_{K, L} a_{K, L} \phi_{k_{1}}^{R} \phi_{k_{2}}^{R} \cdots|R\rangle\langle N S| \phi_{l_{1}}^{N S} \phi_{l_{2}}^{N S} \cdots, \\
K=\left\{k_{i}\right\}, L=\left\{l_{i}\right\}
\end{gathered}
$$

and to calculate the coefficients $a_{K, L}$. After normalizing $\phi_{n}$ suitably to $\varphi_{n}(19,20)$, we see " $a_{K, L} /$ (normalization factor)" are identified with Pfaffians of $X_{k, l}$. With the aid of a relation satisfied by Pfaffian

$$
\omega^{\wedge n}=n ! \operatorname{Pf}\left(b_{i j}\right) x_{1} \wedge x_{2} \cdots \wedge x_{2 n}
$$

where $x_{k}(1 \leq k \leq 2 n)$ is a Grassmann variable and

$$
\omega=\sum_{1 \leq i<j \leq 2 n} b_{i j} x_{i} \wedge x_{j}
$$

we get $(15,16)$.

\footnotetext{
*We are indebted to M.Jimbo for explaining the details of Ref. [6].
} 
Wick's theorem can be generalized to the present situation and we only need to calculate oneand two-point correlation functions for $a_{K, L}$. To calculate these, we rewrite (14) and introduce auxiliary operators

$$
\begin{gathered}
\tilde{\phi}^{N S}(w) \Omega_{R}^{N S}\left(q^{-4}\right)=\Omega_{R}^{N S}\left(q^{-4}\right) \tilde{\phi}^{R}(w), \\
\tilde{\phi}^{N S}(w)=(-1)^{-1 / 2} w^{1 / 2} \frac{\left(q w^{-1} ; q^{4}\right)_{\infty}}{\left(q^{3} w^{-1} ; q^{4}\right)_{\infty}} \phi^{N S}(w), \\
\tilde{\phi}^{R}(w)=\frac{\left(q w ; q^{4}\right)_{\infty}}{\left(q^{3} w ; q^{4}\right)_{\infty}} \phi^{R}(w)=f_{+}(w) \phi^{R}(w),
\end{gathered}
$$

we set $\Omega\left(z=q^{4}\right)$ for simplicity. They are defined to satisfy

$$
\left\langle N S\left|\tilde{\phi}_{n}^{N S}=0(n<0), \quad \tilde{\phi}_{n}^{R}\right| R\right\rangle=0(n>0), \quad \tilde{\phi}_{0}^{R}|R\rangle=|R\rangle,
$$

and this enables us to see that

$$
\left\langle N S\left|\Omega_{R}^{N S}\left(q^{-4}\right) \tilde{\phi}^{R}(z) \tilde{\phi}^{R}(w)\right| N S\right\rangle=\left\langle N S\left|\tilde{\phi}^{N S}(z) \Omega_{R}^{N S}\left(q^{-4}\right) \tilde{\phi}^{R}(w)\right| N S\right\rangle
$$

contains only negative (positive) powers of $z(w)$. On the other hand the expectation value of

$$
\begin{gathered}
\left\{\tilde{\phi}^{R}(z), \tilde{\phi}^{R}(w)\right\}=f_{+}(z) f_{+}(w)\left(\delta\left(\frac{q^{2} w}{z}\right)+\delta\left(\frac{w}{q^{2} z}\right)\right), \\
\delta(z)=\sum_{n \in \mathbb{Z}} z^{n},
\end{gathered}
$$

with respect to $\langle N S| \Omega_{R}^{N S}\left(q^{-4}\right)$ and $|R\rangle$ is

$$
\begin{gathered}
\left\langle N S\left|\Omega_{R}^{N S}\left(q^{-4}\right) \tilde{\phi}^{R}(z) \tilde{\phi}^{R}(w)\right| N S\right\rangle+\left\langle N S\left|\Omega_{R}^{N S}\left(q^{-4}\right) \tilde{\phi}^{R}(w) \tilde{\phi}^{R}(z)\right| N S\right\rangle \\
=f_{+}(z) f_{+}(w)\left(\delta\left(\frac{q^{2} w}{z}\right)+\delta\left(\frac{w}{q^{2} z}\right)\right)
\end{gathered}
$$

where we normalize $\left\langle N S\left|\Omega_{R}^{N S}\left(q^{-4}\right)\right| R\right\rangle=1$. And we get

$$
\left\langle N S\left|\Omega_{R}^{N S}\left(q^{-4}\right) \tilde{\phi}^{R}(z) \tilde{\phi}^{R}(w)\right| R\right\rangle=\frac{1-q w}{1-q^{2} w / z}+\frac{1-q^{-1} w}{1-q^{-2} w / z}-1
$$

Expanding the last line of the following equation as in 9

$$
\begin{gathered}
\left\langle N S\left|\Omega_{R}^{N S}\left(q^{-4}\right) \phi^{R}(z) \phi^{R}(w)\right| R\right\rangle=\sum_{n, m \in \mathbb{Z}}\left\langle N S\left|\Omega_{R}^{N S}\left(q^{-4}\right) \phi_{n}^{R} \phi_{m}^{R}\right| R\right\rangle z^{-n} w^{-m} \\
=\frac{1}{f_{+}(z) f_{+}(w)}\left\{\frac{1-q w}{1-q^{2} w / z}+\frac{1-q^{-1} w}{1-q^{-2} w / z}-1\right\},
\end{gathered}
$$

we have

$$
\left\langle N S\left|\Omega_{R}^{N S}\left(q^{-4}\right) \phi_{-n}^{R} \phi_{-m}^{R}\right| R\right\rangle=X_{m, n} \gamma_{n} \gamma_{m} q^{n+m}(n, m \geq 0)
$$


Similar calculation yields

$$
\begin{gathered}
\left\langle N S\left|\phi_{k+1 / 2}^{N S} \Omega_{R}^{N S}\left(q^{-4}\right) \phi_{-n}^{R}\right| R\right\rangle=-(-1)^{1 / 2} X_{-k-1 / 2, n} \gamma_{n} \gamma_{k} q^{n+k}(n, k \geq 0), \\
\left\langle N S\left|\phi_{k+1 / 2}^{N S} \phi_{l+1 / 2}^{N S} \Omega_{R}^{N S}\left(q^{-4}\right)\right| R\right\rangle=-X_{l+1 / 2, k+1 / 2} \gamma_{l} \gamma_{k} q^{l+k}(k, l \geq 0) .
\end{gathered}
$$

$z$-dependence of $\Omega_{N S}^{R}(z)$ is recovered with the equation

$$
\begin{gathered}
\zeta^{d^{R}} \Omega_{N S}^{R}(z) \zeta^{-d^{N S}}=\Omega_{N S}^{R}\left(\zeta^{-1} z\right), \\
\zeta^{-d^{i}} \phi^{i}(z) \zeta^{d^{i}}=\phi^{i}(\zeta z), \\
\left\langle i\left|d^{i}=d^{i}\right| i\right\rangle=0,
\end{gathered}
$$

where $d^{i}$ 's are the fermionic part of $d$ of (3)

$$
d^{i}=-\sum_{k>0} k N_{k}^{\phi^{i}},(i=N S \text { or } R)
$$

and satisfy

$$
\left[d^{i}, \phi_{n}^{i}\right]=n \phi_{n}
$$

To derive (57), we multiply (14) by $\zeta^{d^{R}}, \zeta^{-d^{N S}}$ from left and right respectively.

\section{Acknowledgement}

The author thanks M.Jimbo, H.Konno, S.Odake and J.Shiraishi for helpful discussions. He also thanks A.Kuniba for warm encourragement.

\section{Appendix A. BOSON}

Followings are useful formulae for normal ordering bosons. We set $(z)_{\infty}=\left(z ; q^{4}\right)_{\infty}$ for brevity.

$$
\begin{aligned}
B_{I,>}(z) E_{<}^{-}(w) & =\frac{(q w / z)_{\infty}}{\left(q^{-1} w / z\right)_{\infty}} E_{<}^{-}(w) B_{I,>}(z), \\
E_{>}^{-}(w) B_{I,<}(z) & =\frac{\left(q^{9} z / w\right)_{\infty}}{\left(q^{7} z / w\right)_{\infty}} B_{I,<}(z) E_{>}^{-}(w), \\
B_{I,>}(z) E_{<}^{+}(w) & =\frac{\left(q^{-3} w / z\right)_{\infty}}{\left(q^{-1} w / z\right)_{\infty}} E_{<}^{+}(w) B_{I,>}(z), \\
E_{>}^{+}(w) B_{I,<}(z) & =\frac{\left(q^{5} z / w\right)_{\infty}}{\left(q^{7} z / w\right)_{\infty}} B_{I,<}(z) E_{>}^{+}(w), \\
B_{I I,>}(z) E_{<}^{+}(w) & =\frac{\left(q^{-1} w / z\right)_{\infty}}{\left(q^{-3} w / z\right)_{\infty}} E_{<}^{+}(w) B_{I I,>}(z), \\
E_{>}^{+}(w) B_{I I,<}(z) & =\frac{\left(q^{3} z / w\right)_{\infty}}{(q z / w)_{\infty}} B_{I I,<}(z) E_{>}^{+}(w),
\end{aligned}
$$




$$
\begin{aligned}
B_{I I,>}(z) E_{<}^{-}(w) & =\frac{\left(q^{-1} w / z\right)_{\infty}}{(q w / z)_{\infty}} E_{<}^{-}(w) B_{I I,>}(z), \\
E_{>}^{-}(w) B_{I I,<}(z) & =\frac{\left(q^{3} z / w\right)_{\infty}}{\left(q^{5} z / w\right)_{\infty}} B_{I I,<}(z) E_{>}^{-}(w), \\
F_{I I,>}(z) E_{<}^{-}(w) & =\left(1-\frac{w}{q^{2} z}\right) E_{<}^{-}(w) F_{I I,>}(z), \\
E_{>}^{-}(w) F_{I I,<}(z) & =\left(1-\frac{q^{2} z}{w}\right) F_{I I,<}(z) E_{>}^{-}(w), \\
F_{I I,>}(z) E_{<}^{+}(w) & =\frac{1}{1-q^{-4} w / z} E_{<}^{+}(w) F_{I I,>}(z), \\
E_{>}^{+}(w) F_{I I,<}(z) & =\frac{1}{1-z / w} F_{I I,<}(z) E_{>}^{-}(w), \\
E_{>}^{-}\left(w_{1}\right) E_{<}^{+}\left(w_{2}\right) & =\frac{1}{1-w_{2} / w_{1}} E_{<}^{+}\left(w_{2}\right) E_{>}^{-}\left(w_{1}\right), \\
E_{>}^{+}\left(w_{2}\right) E_{<}^{-}\left(w_{1}\right) & =\frac{1}{1-w_{1} / w_{2}} E_{<}^{-}\left(w_{1}\right) E_{>}^{+}\left(w_{2}\right),
\end{aligned}
$$

\section{APPENDIX B. FERMION}

For $\Omega_{R}^{N S}(z)$, we show the equations corresponding to the ones from (51) to (56)

$$
\begin{gathered}
\tilde{\phi}^{R^{\prime}}(w) \Omega_{N S}^{R}\left(q^{-4}\right)=\Omega_{N S}^{R}\left(q^{-4}\right) \tilde{\phi}^{N S^{\prime}}(w), \\
\tilde{\phi}^{R^{\prime}}(w)=\frac{\left(q / w ; q^{4}\right)_{\infty}}{\left(q^{3} / w ; q^{4}\right)_{\infty}} \phi^{R}(w) \\
\tilde{\phi}^{N S^{\prime}}(w)=(-1)^{1 / 2} w^{-1 / 2} \frac{\left(q w ; q^{4}\right)_{\infty}}{\left(q^{3} w ; q^{4}\right)_{\infty}} \phi^{N S}(w), \\
\langle R| \tilde{\phi}_{n}^{R^{\prime}}=0(n<0), \quad\langle R| \tilde{\phi}_{0}^{R^{\prime}}=\left\langle R\left|, \quad \tilde{\phi}_{n}^{N S^{\prime}}\right| N S\right\rangle=0(n>0), \\
\left\langle R\left|\tilde{\phi}^{R^{\prime}}(z) \tilde{\phi}^{R^{\prime}}(w) \Omega_{N S}^{R}\left(q^{-4}\right)\right| N S\right\rangle=\frac{1-q / z}{1-q^{2} w / z}+\frac{1-q^{-1} / z}{1-q^{-2} w / z}-1 \\
\left\langle R\left|\phi_{n}^{R} \phi_{m}^{R} \Omega_{N S}^{R}\left(q^{-4}\right)\right| N S\right\rangle=X_{n, m} \gamma_{n} \gamma_{m} q^{n+m}(n, m \geq 0), \\
\left\langle R\left|\phi_{n}^{R} \Omega_{N S}^{R}\left(q^{-4}\right) \phi_{-k-1 / 2}^{N S}\right| N S\right\rangle=(-1)^{1 / 2} X_{-k-1 / 2, n} \gamma_{n} \gamma_{k} q^{n+k}(n, k \geq 0), \\
\left\langle R\left|\Omega_{N S}^{R}\left(q^{-4}\right) \phi_{-k-1 / 2}^{N S} \phi_{-l-1 / 2}^{N S}\right| N S\right\rangle=X_{l+1 / 2, k+1 / 2} \gamma_{l} \gamma_{k} q^{l+k}(k, l \geq 0)
\end{gathered}
$$




\section{Appendix C. Calculation of Eqn.(54)}

We show details of calculation of (54). From (21)

$$
\begin{aligned}
& \left\langle N S\left|\Omega_{R}^{N S}\left(q^{-4}\right) \phi^{R}(z) \phi^{R}(w)\right| R\right\rangle \\
& =\frac{1}{f_{+}(z) f_{+}(w)}\left\{\frac{1-q w}{1-q^{2} w / z}+\frac{1-q^{-1} w}{1-q^{-2} w / z}-1\right\} \\
& =\sum_{k \geqslant 0, l \geqslant 0} \gamma_{k}(q z)^{k} \gamma_{l}(q w)^{l}\left\{\sum_{a \geqslant 0}\left((1-q w)\left(\frac{q^{2} w}{z}\right)^{a}+(1-w / q)\left(\frac{w}{q^{2} z}\right)^{a}\right)-1\right\} \\
& =\sum_{0 \leqslant a \leqslant m} \gamma_{n+a} \gamma_{m-a} \eta_{a} q^{n+m} z^{n} w^{m}-\sum_{0 \leqslant a \leqslant m-1} \gamma_{n+a} \gamma_{m-a-1}\left(q^{2 a}+q^{-2(a+1)}\right) q^{n+m} z^{n} w^{m}-\gamma_{n} \gamma_{m} z^{n} w^{m}
\end{aligned}
$$

Hence the equation to be proved is

$$
X_{n, m} \gamma_{n} \gamma_{m}=\sum_{0 \leqslant a \leqslant m} \gamma_{n} \gamma_{m} \eta_{a}-\sum_{0 \leqslant a \leqslant m-1} \gamma_{n+a} \gamma_{m-a-1}\left(q^{2 a}+q^{-2(a+1)}\right)-\gamma_{n} \gamma_{m} z^{n} w^{m},
$$

which is equivalent to

$$
X_{n, m}=1+\left(1-t^{-1}\right)\left(1+t^{2 n}\right) \sum_{1 \leqslant a \leqslant m} \frac{\left(t^{1+2 n} ; t^{2}\right)_{a-1}}{\left(t^{2+2 n} ; t^{2}\right)_{a-1}} \frac{\left(t^{2 m-2 a+2} ; t^{2}\right)_{a}}{\left(t^{2 m-2 a+1} ; t^{2}\right)_{a}} \frac{t^{a}}{1-t^{2(n+a)}}
$$

where we set $t=q^{2}$. It can be proved by induction with respect to $k$ that the summation over $a=m, m-1, \cdots, m-k$ yields

$$
t^{m-k} \frac{\left(t^{1+2 n} ; t^{2}\right)_{m-k-1}}{\left(t^{2+2 n} ; t^{2}\right)_{m-k-1}} \frac{\left(t^{2 k+2} ; t^{2}\right)_{m-k}}{\left(t^{2 k+1} ; t^{2}\right)_{m-k}} \frac{\sum_{j=0}^{k} t^{2 j}}{1-t^{2(n+k)}} .
$$

Setting $k=m-1$ we can see that the right hand side of (61) is equal to $\frac{t^{2 m}-t^{2 n}}{1-t^{2(n+m)}}$.

\section{REFERENCES}

[1] B.Davies, O.Foda, M.Jimbo, T.Miwa and A.Nakayashiki, Diagonalization of the XXZ Hamiltonian by vertex operators, Commun. Math. Phys. 151, 89 (1993).

[2] M.Jimbo, K.Miki, T.Miwa and A.Nakayashiki, Correlation functions of the XXZ model for $\Delta<-1$, Phys.Lett.A, 168, 256 (1992).

[3] M.Jimbo, T.Miwa, Algebraic Analysis of Solvable Lattice Models, American Mathematical Society, 1993.

[4] M.Idzumi, Level two irreducible representations of $U_{q}(\widehat{s l}(2))$, vertex operators and their correlations, Int.J.Mod.Phys. A9, 4449 (1994).

[5] M.Idzumi, Correlation functions of the spin-1 analog of the XXZ model, hep-th/9307129.

[6] O.Foda, M.Jimbo, T.Miwa, K.Miki and A.Nakayashiki, Vertex operators in solvable lattice models, J.Math.Phys. 35, 13 (1994).

[7] M.Jimbo and J.Shiraishi, A Coset-type construction for the deformed Virasoro algebra, Lett. Math. Phys. 43, 173 (1998).

[8] I.B.Frenkel, N.Yu.Reshetikhin, Quantum affine algebras and holonomic difference equations, Commun.Math.Phys. 146, 1 (1992). 
[9] E.Date, M.Jimbo, M.Okado, Crystal base and q-vertex operators, Commun.Math.Phys. 155,47 (1993).

[10] D.Friedan, Z.Qiu and S.Shenker, Superconformal invariance in two dimensions and the tricritical Ising model, Phys.Lett.B 151, 37 (1985); E.F.Corrigan and D.I.Olive, Fermion-meson vertices in dual theories, Nuovo Cim. 11A, 749 (1972); E.F.Corrigan and P.Goddard, Gauge conditions in the dual fermion model, Nuovo Cim. 18A, 339 (1973); M.Kato and S.Matsuda, Null Field Construction in Conformal and Superconformal Algebras, Adv.Std.Pure Math. 16, 205 (1988).

[11] V.G.Drinfeld, A new realization of Yangians and quantized affine algebras, Soviet Math. Doklady 36, 212 (1988).

[12] V.Chari and A.Pressley, Quantum Affine Algebra, Commun. Math. Phys. 142, 261 (1991).

Institute of Physics, Graduate School of Arts and Sciences, University of Tokyo, Tokyo 153, JAPAN

E-mail address: ss77070@komaba.ecc.u-tokyo.ac.jp 